\title{
The generation revenue and demand payment assessment for pool based market model in Malaysia electricity supply industry
}

\author{
Zuraidah Ngadiron', N. H. Radzi², M. Y. Hassan $^{3}$ \\ ${ }^{1,2}$ Green and Sustainable Energy (GSEnergy) Focus Group, Faculty of Electrical and Electronic Engineering, University \\ Tun Hussien Onn Malaysia, Malaysia \\ ${ }^{3}$ Centre of Electrical Energy Systems (CEES), Faculty of Electrical Engineering, University Teknologi
}

Malaysia, Malaysia

\section{Article Info}

Article history:

Received Mar 29, 2019

Revised May 4, 2019

Accepted Jun 26, 2019

\section{Keywords:}

Capacity payment

Efficiency

Electricity supply industry

Pool market

Spot market

\begin{abstract}
The objective of this paper is to address the economic benefits in term of generation revenue and demand payment for the pool based market model in Malaysia electricity supply industry (MESI). In pool market model, there are issues on the benefit of the generators such as too high system marginal price (SMP) during peak demand and no revenue during low demand. Therefore, conceptual study for two bus test system in MESI involving four generators around Peninsular Malaysia is conducted to perform the economic analysis in term of generation revenue and demand assessment considering existing single buyer model and pool based market model, i.e., pool model, spot market model and the proposed model, in order to identify which market model is superior. As a result, the proposed model managed to decrease the demand payment as it is proportional to generation revenue, even though the generation revenue is at intermediate value and succeed to increase the low and medium generator's revenue.
\end{abstract}

Copyright (C) 2019 Institute of Advanced Engineering and Science. All rights reserved.

\section{Corresponding Author:}

Zuraidah Ngadiron, Green and Sustainable Energy (GSEnergy) Focus Group,

Faculty of Electrical and Electronic Engineering,

Universiti Tun Hussien Onn Malaysia, 86400 Parit Raja, Batu Pahat, Johor, Malaysia.

Email: zuraidahn@gmail.com

\section{INTRODUCTION}

The historical evolution of Malaysian Electricity Supply Industry (MESI) leads to the formalization of the single buyer. Worldwide experience has raised concern on the single buyer model as it is lack of transparency and fairness, poor system planning and non-competitive procurement which are some of the drawbacks due to poorly constructed [1]. Several observers citing conflict of interest, duplication of cost and tariff hikes and these concerns can be mitigated as long as the single buyer remains regulated [2]. MESI continues to face multi-dimensional challenges in term of tightness in fuel supply, industry governance and unsustainable tariff structure [3]. Therefore, more reform initiatives for MESI are expected to be implemented in the near future. Hence, the pool market model is the most preferred electricity market model. The discrepancies for the single buyer model, pool market model, spot market model, and the proposed model will be explained in next section.

The aim of this research study is to improve the pool based market model which useful for MESI, in order to enhance efficiency and to promote competition in order to lower costs. The objective is to identify which market model is superior using two bus test system in MESI involving four generators around Peninsular Malaysia with combined cycle gas turbine (CCGT) and thermal power plants. Consequently, the findings can be applied in assisting and creating new policy set out for better electricity market model as; 
a. Single buyer model

There are two types of payment in single buyer model applied by MESI, which are energy payment and capacity payment bound under the Power Purchased Agreements (PPA) [4]. For energy payment, Tenaga Nasional Berhad (TNB) will only pay for the amount of the energy it took from the Independent Power Producers (IPP) [5, 6]. Meanwhile, the capacity payment is the amount of payment which TNB has to pay regardless whether the generated power is used or not and it is mean for the IPP to recover a portion of its investment [7]. The role for this payment is to provide extra revenue to the generator which are not generated through the charges of energy price. However, the energy price is the key of the pattern dispatch. Ideally, only the cheaper generator or lowest running cost generator should be generating power to meet load demand.

b. Pool market model

The pool market model pricing scheme can be applied into two types; uniform price which based on the system marginal price, and pay as bid which is based on the generator's energy bid price [8]. The final price paid to the generators is a combination of system marginal price (SMP), loss of load probability (LOLP) and the value of lost load (VOLL) [9]. As the pure pool does not provide capacity payment to the generator, during low load demand, expensive generators may expose to losses due to not being selected to supply electricity [10]. For the pure pool market, the equation for pool purchased price, PPP is only based on the SMP [11].

c. Spot market model

The electricity market in Australia is based on the energy only market design. The pool is managed by the Australian Energy Market Operator (AEMO) [12, 13]. AEMO issues dispatch instructions to generators at five-minute intervals throughout each day based on the offers generators have submitted in the bidding process [14]. The electricity is traded in a constrained real time spot market where prices are set each 5 minutes by AEMO [15]. The generators submit offers every five minutes and a dispatch price is determined every five minutes. The final price is determined as the six dispatch prices are averaged every half-hour to determine the spot price for each trading interval for each of the regions of the Australian National Electricity Market (NEM) [16]. AEMO uses the spot price as the basis for the settlement of financial transactions for all energy traded in the NEM [17]. There are 288 dispatch intervals every day [16]. The dispatch price represents the cost to supply the last megawatt of electricity to meet demand, and applies to all generators scheduled into production regardless of the level of their original offer. A trading interval in the NEM is a half-hour period [18]. Hence, there are 48 trading intervals in the market each day. The spot price of electricity for all 30-minute trading intervals each day is the average of the six dispatch prices during the preceding half-hour.

d. The proposed model

The proposed model apply the approaches of the minimum generation capacity payment mechanism, and base load demand sharing involving the efficiency of the generators [19]. These approaches are to educate the generators to apply low prices for electricity generated, so that the IPPs can contribute power to the power purchaser at all times [20-22]. The proposed model has categorised the electricity demand into two areas, high demand area and low demand area which determined from the daily electricity demand curve. A line is drawn on the hourly electricity demand curve at $80 \%$ of the peak demand as the reference line to distinguish the low demand, and high demand areas [19]. The low demand area consists of the hourly electricity demand below the $80 \%$ of the peak demand value and will be traded through bidding competition as in the pool market plus with the minimum generation capacity payment [23]. However, the $80 \%$ reference line will separate electricity demand in the high area into two parts [19]. First, the electricity demand below the $80 \%$ reference line will be traded equally among the base load plants through demand sharing approach. Second, all IPPs will compete against each other to supply the remaining demand in the area above the $80 \%$ reference line in pool market based on energy price [23].

\section{RESEARCH METHOD}

Figure 1 illustrated the two bus system to provide an understanding on the basic concept of proposed approach. The case study is carried out by calculating the generation revenue for four generators around Peninsular Malaysia. The analysis of the power generation revenue is conducted at low load demand (1500 MW), medium demand (3250 MW) and high demand (5000 MW) to acquire the results of generator's revenue for pool based market model. Four criteria are taken into accounts; the available capacity for each generator in megawatt (MW), the capacity price in $\mathrm{RM} / \mathrm{kW} / \mathrm{month}$, energy price in RM/MWh and efficiency, $\eta$, as shown in Table 1. Since, the monetary values involved in the study are confidential; therefore estimated values are being used instead. 


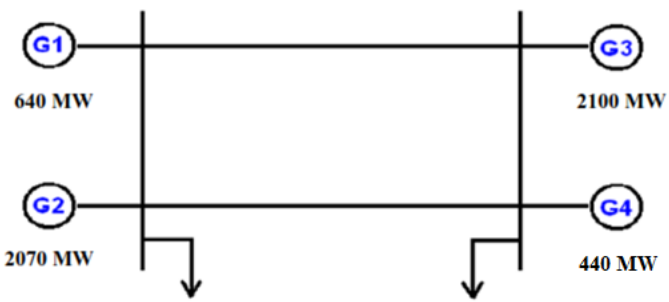

Figure 1. Four generators with two loads

Table 1. The details of each IPP in 2-bus Malaysia electricity system

\begin{tabular}{cccccc}
\hline Gen. & Plant Type & Available Capacity (MW) & $\begin{array}{c}\text { Capacity Price } \\
(\text { RM/MWh/month) }\end{array}$ & $\begin{array}{c}\text { Energy Price } \\
\text { (RM/MWh) }\end{array}$ & Efficiency, $\eta(\%)$ \\
\hline G1 & CCGT & 650 & 35000 & 155 & 43.64 \\
G2 & Thermal & 2070 & 30000 & 160 & 20.91 \\
G3 & Thermal & 2100 & 55000 & 170 & 25.82 \\
G4 & CCGT & 440 & 30000 & 200 & 43.64 \\
\hline
\end{tabular}

The purpose is to detail out the explanations towards the trading in pool based market model, in order to see the effect of load variation towards the generator's revenue, total generation revenue and demand payment. Figure 2 shows the aggregated generation curve for the bidding process. The transmission network is assumed to be lossless. The available capacities in MW for each of these busses are being used to acquire all the results for generation revenue. Only combine cycle gas turbine (CCGT) and thermal plant type are chosen due to the efficiency and price offered by the generator. This is because the generation costs of open cycle gas turbine (OCGT) are much higher. However, the main reason for the OCGT high generation cost is the low load-factor of the peak-load services [24, 25]. From the results, a proper and depth analysis was discussed.

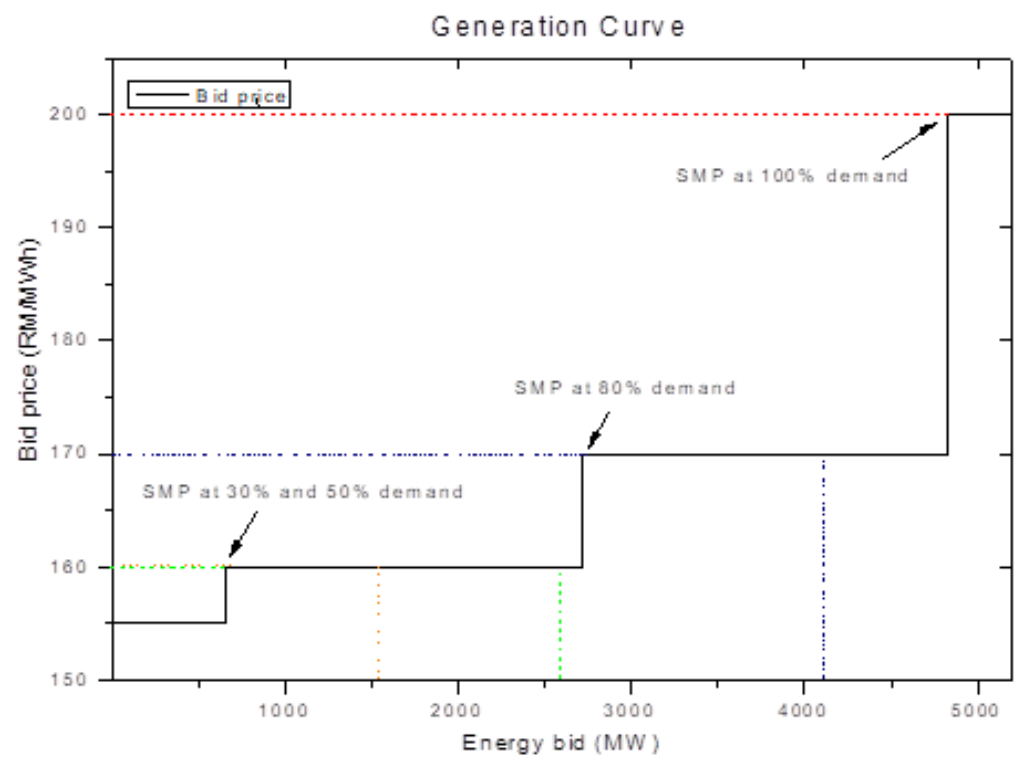

Figure 2. The aggregated generation curve and the SMP at 30\%, 50\%, $80 \%$ and $100 \%$

\section{RESULTS AND ANALYSIS}

Four generators with two loads that supply $1500 \mathrm{MW}, 3250 \mathrm{MW}$, and $5000 \mathrm{MW}$ demand is used in this case study. The comparison have been done between the single buyer model, pool model, spot market model and the proposed model in order to see the variation of economic benefit in term of IPP's generation revenue, which is represented by the generators, total generation revenue based on demand, and investigation 
on demand payment to analysis the payment by energy's buyer to the power producer according to the demand. Based on the analysis of the electricity demand values in MW, there are four categories; $30 \%$ demand, $50 \%$ demand, $80 \%$ demand, and $100 \%$ demand as shown in Table 2 . In order to be chosen in the generation dispatch, IPPs must win the energy bidding. The IPPs with lower energy bid price contrasted with the hourly SMP will be chosen to satisfy the power demand for that hour. The SMP at specific hour is determined when the load curve and supply curve intersect as shown in Figure 2. Thus, the SMP determined the payment for all in-merit generators. The SMP at 100\% demand was RM 200 followed by RM 170 for $80 \%$ demand, and RM 160 for 50\% and 30\% demand respectively. These SMP will be used to determine the pool purchased price. All participated IPPs in the electricity dispatch will be paid according to the pool purchased price which is determined from hourly SMP, rather than the initial bid. Therefore, the nonparticipated IPPs are exposed to the risk of losing their revenue, because only at $100 \%$ demand, all IPPs received the revenue. This means that more IPPs will lose their revenue as the electricity demand keeps reducing, but instead the IPPs revenue in the pool market continues to strike as the demand keeps increasing due to most IPPs are paid at higher price compared to their own bid price. For example, IPP 1 is paid with RM 200 for every MW power produced at 100\% demand, which is RM 45 higher compared to its initial bid price. Therefore, in the proposed model, power contribution is chosen at $80 \%$ for demand sharing which the resulting SMP, RM 170 will make the production cost of electricity will be lower, in order to control the price fluctuation during high demand. The analysis in term of economic benefit will be carried out to see the performance of IPP's generation revenue, total generation revenue and investigation on demand side regarding the payment by the purchasers to the power producers.

Table 2. Summarised of the SMP according to demand

\begin{tabular}{cccccc}
\hline \multirow{2}{*}{ Gen. } & Energy Price & \multicolumn{4}{c}{ SMP (RM) with Demand Contribution at } \\
\cline { 3 - 6 } & $(\mathrm{RM} / \mathrm{MWh})$ & $1500 \mathrm{MW}(30 \%)$ & $2500 \mathrm{MW}(50 \%)$ & $4000 \mathrm{MW}(80 \%)$ & $5000 \mathrm{MW}(100 \%)$ \\
\hline G1 & 155 & 160 & 160 & 170 & 200 \\
G2 & 160 & 160 & 160 & 170 & 200 \\
G3 & 170 & 160 & 160 & 170 & 200 \\
G4 & 200 & 160 & 160 & 170 & 200 \\
\hline
\end{tabular}

The applied values of capacity price for the generators are differ from one another because of different mechanism and approaches to produce electric power such as fuel type, and the distance of the plant to the transmission. Even though, the energy payment can cover the variable costs such as labour cost, maintenance cost, and fuel cost, significantly, inadequate capacity payment reduces the generator ability to cover its fixed costs and will lead to poor performances. The importance of capacity payment mechanisms in electricity markets is to ensure security of electricity supply, and to fill the "missing-money" gap. In MESI, some of the IPPs have covered their fixed cost in ten years. As the PPAs are applied for 21 years, full capacity payment still continuously paid which caused financial lost to the energy buyers. Therefore, the purposed of the new mechanism for capacity payment is to solve the capacity payment issue. Instead of paying full capacity payment to the generator, minimum generation capacity payment based on generator's efficiency is introduced as compensation because the possibility of expensive generators for not being selected in the bidding competition is high, so that the IPPs can covers their variable cost. Meanwhile, the IPPs which have won in the bidding competition also will be given this same type of capacity payment as an incentive. Significantly, this incentive as a reward in order to educate the IPPs to bid, and sell their electricity produced at lower price. Furthermore, the IPPs are able to compete for more dispatch, and gain more revenue.

\subsection{Generation revenue}

To illustrate more details, Figure 3 shows the generation revenue for each generator during low, medium and high demand for Gen 1 and Gen 2. The generators represented the IPPs involved in the bidding competition. Meanwhile, generation revenue clarifies the payment received by the IPPs from the generated energy and the market models represent the different types of market model used in the comparison.For single buyer, during all demand, Gen 1 and Gen 2 received full payment from energy and capacity payment. Meanwhile, the proposed model, generation revenue for Gen 1 and Gen 2 are increased during low and medium demand compared to pool and spot market models due to improvement in capacity payment which fulfill the requirement in term of generator's efficiency and cost. Gen 1 and Gen 2 for pool and spot market models show the same pattern during low and medium demand, but generation revenue for the spot market slightly lower compared to pool model because of spot prices were taken from the average of dispatch prices in half an hour. 


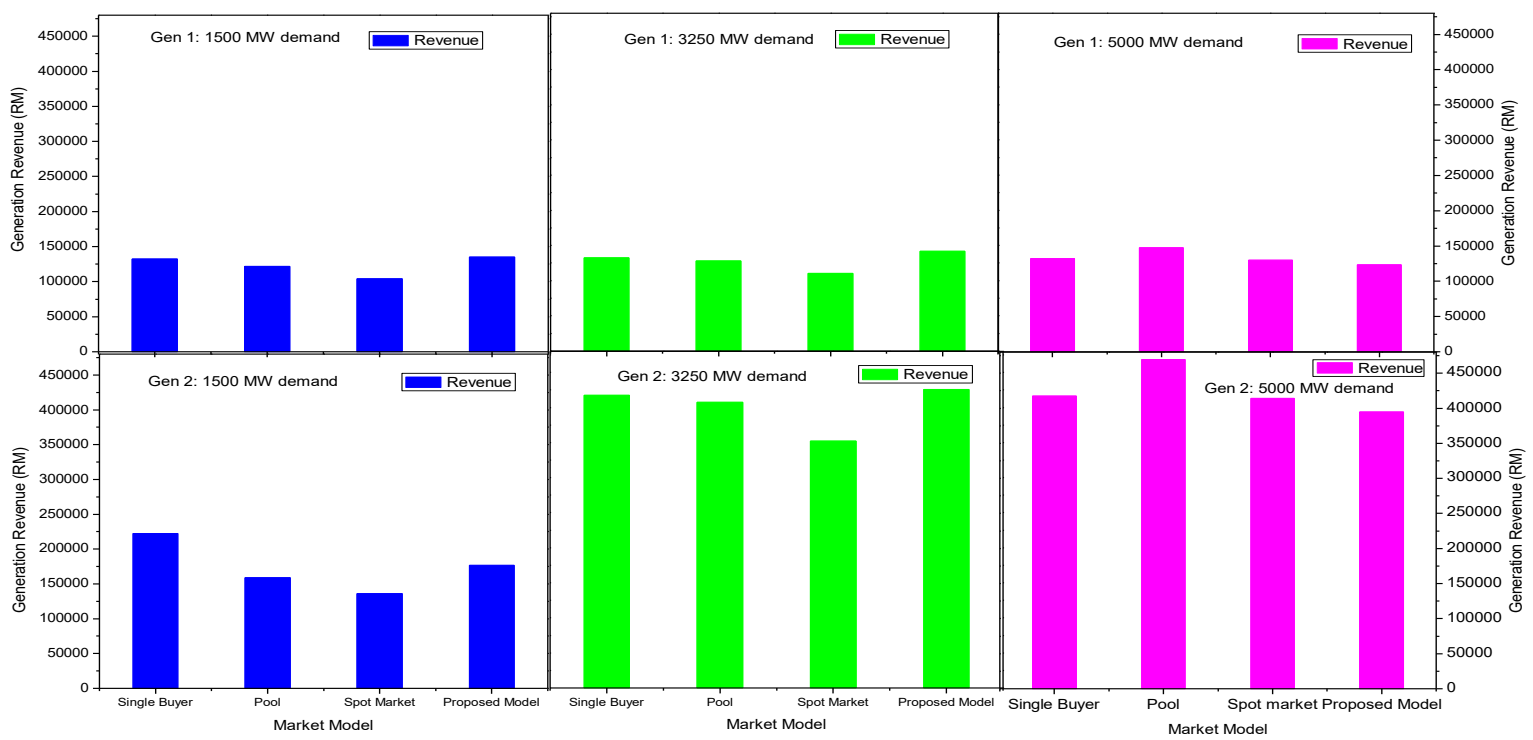

Figure 3. Comparison of Gen 1 and Gen 2 revenue for low, medium and high demand

Figure 4 depicts the generator's revenue for Gen 3 and Gen 4 during low to high demand. According to single buyer model, the generation revenue for Gen 3 continued to increase compared to others market models, while no revenue for Gen 3 and Gen 4 for pool and spot market models during low demand. This shows the weaknesses of pool market, where too high SMP during peak demand and no revenue during low demand. During medium demand, Gen 3 shows that the pool and spot market models revenue increased due to demand increase, which opens chances for expensive generators to be selected in the bidding competition. The revenue for proposed market is increased compared to the pool and spot market models by $39.68 \%$ and $61.81 \%$ respectively due to the improvement in capacity payment. The revenue for pool model increased when SMP is high during peak demand. The revenue for the proposed model decreased compared to pool model by $15.21 \%$ due to base load sharing approach and pool trading during high demand.

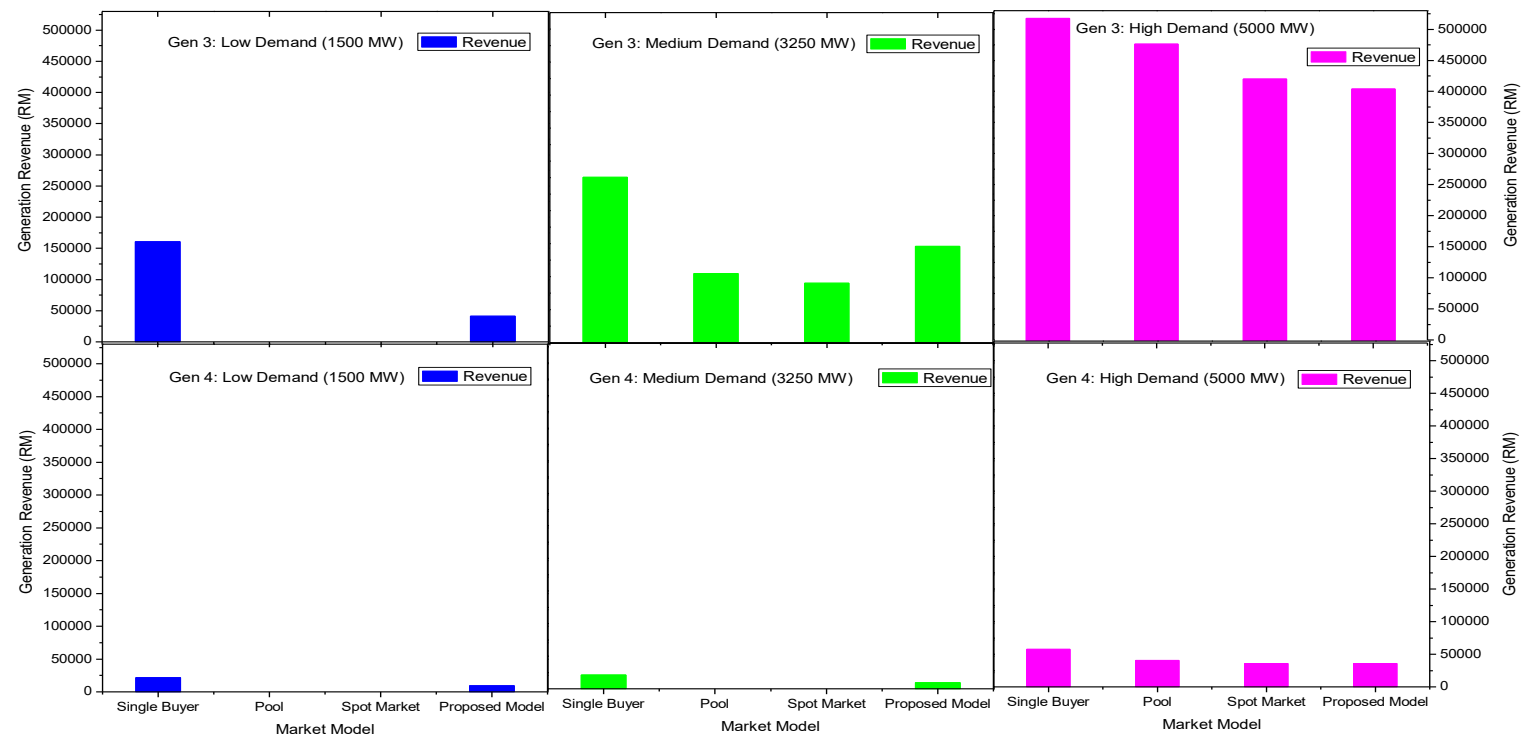

Figure 4. Comparison of Gen 3 and Gen 4 revenue for low, medium and high demand

There was no revenue during low and medium demand for Gen 4, which is categorised as an expensive generator for pool and spot market models due to the pattern dispatch, where the generators are 
ranked due to energy price, from the lowest price to the highest price. Thus, the cheapest generator should be generating to meet demand, causing the expensive generator to lose in the bidding competition. Meanwhile, there was no energy payment for the single buyer model during low and medium demand and the revenue only depended on the full capacity payment. Even though there was no energy usage during that moment, the purchaser still needed to bear the expenses due to full capacity payment. However, the proposed model managed to gain revenue due to minimum generation capacity payment which was given as compensation and stayed online when electricity was needed. During high demand, the proposed model still managed to receive the revenue for the expensive generator due to pool trading for the remaining demand from the base load sharing. Table 3 shows the total result of each generator's revenue, which represent the IPPs.

Table 3. IPP's generation revenue

\begin{tabular}{ccccc}
\hline \multicolumn{4}{c}{ Total Revenue (RM) } \\
\hline Gen & Single Buyer & Pool & Spot Market & Proposed Method \\
G1 & $397,041.67$ & $396,980.82$ & $344,500.00$ & $400,475.48$ \\
G2 & $1,057,150.00$ & $1,036,141.37$ & $901,900.00$ & $997,275.54$ \\
G3 & $928,350.00$ & $580,757.26$ & $510,100.00$ & $591,147.44$ \\
G4 & $100,166.67$ & $40,832.88$ & $36,000.00$ & $54,668.22$ \\
\hline
\end{tabular}

Figure 5 illustrates the comparison of the total generator's revenue for single buyer, pool, spot market and the proposed models. The generation revenue from Gen 1 until Gen 4 for single buyer is the highest due to energy and capacity payment, even though most of the Gen 3 and Gen 4 did not receive any energy payment during low and medium demand. The spot market has similar characteristics to the pool model, but the revenue is slightly lower compared to the pool model because the energy is paid according to spot prices. The SMP for pool model is varied due to the demand of a certain period. Under the pool model, the revenues for Gen 1 to Gen 3 are slightly higher compared to the expensive generator, Gen 4, because the generators were stacked from the lowest to the highest prices offered. Therefore, the cheaper generators obtained more chances to win the bidding competition.

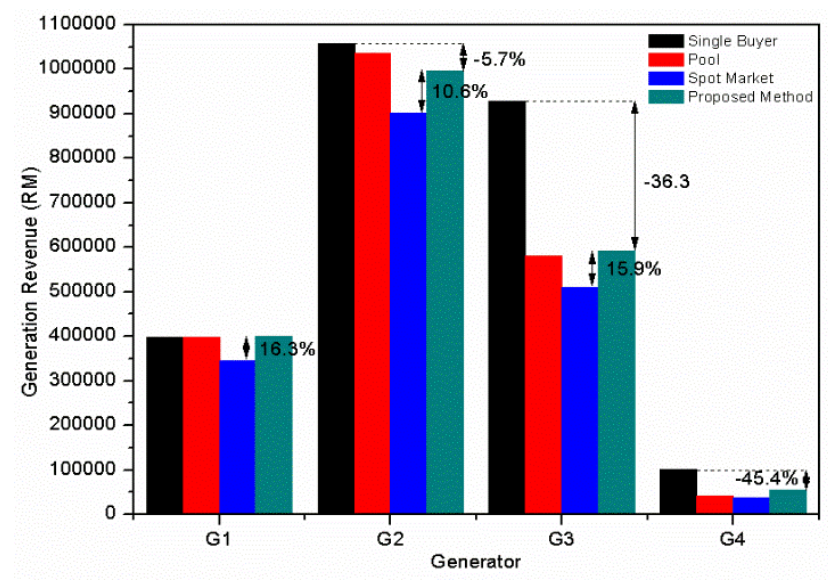

Figure 5. Comparison of total generator's revenue according to market model

Furthermore, during high demand, the low cost generators received excessive revenue due to high SMP. Compared to the spot market model, the generator's revenue for Gen 1 to Gen 3 of the proposed model is higher due to the minimum generation capacity payment and base load sharing approaches. These adding properties reduce the revenue for the expensive generators and lessen the power of bigger capacity generators in order to assure a worthy generation of revenue for all generators as a producer, and a worthy payment for the purchasers. The proposed model guarantees the benefit of all generators regardless of the variation of electricity demand. This will help to educate the generators to bid at cheaper prices for more revenue. It is reasonable that the efficiency of the generator will limit the capacity payment as it describes the real ability and performance of the generator.

Table 4 shows the detailed result of total generation revenue based on demand. From the result, the total revenue for the proposed model is lower compared to the single buyer and pool models which benefit the energy buyers as the value of total revenue for the proposed model is intermediate and will decrease the 
demand payment. Meanwhile, Figure 6 shows the comparison of total revenue generation based on demand according to the types of market models. The single buyer model received the highest revenue for all types of demand. During low demand, the revenue for the proposed model was higher by $29.45 \%$ compared to the pool model due to the implementation of minimum generation capacity payment. However, the pool model indicates the highest generation revenue during peak demand due to high SMP. The revenue for spot market model also exceeded the proposed model during peak demand. This is because when the demand increased, the dispatch price also increased which influenced the spot price. Meanwhile, the generation revenue for the proposed model is lower due to only one SMP during the base load sharing at high demand. The total generation revenue for the proposed model improved by $0.54 \%,-14 \%$ and $17.69 \%$ compared to the pool, spot market and single buyer model, respectively.

Table 4. Generation revenue based on demand

\begin{tabular}{ccccc}
\hline & \multicolumn{4}{c}{ Total Revenue (RM) } \\
\hline Demand (MW) & Single Buyer & Pool & Spot Market & Proposed Method \\
\hline 1500 & $536,402.78$ & $280,438.36$ & $240,000.00$ & $363,015.95$ \\
3250 & $821,702.78$ & $640,027.40$ & $552,500.00$ & $722,604.99$ \\
5000 & $1,124,602.78$ & $1,134,246.58$ & $1,000,000.00$ & $957,945.74$ \\
Total & $2,482,708.33$ & $2,054,712.33$ & $1,792,500.00$ & $2,043,566.68$ \\
\hline
\end{tabular}

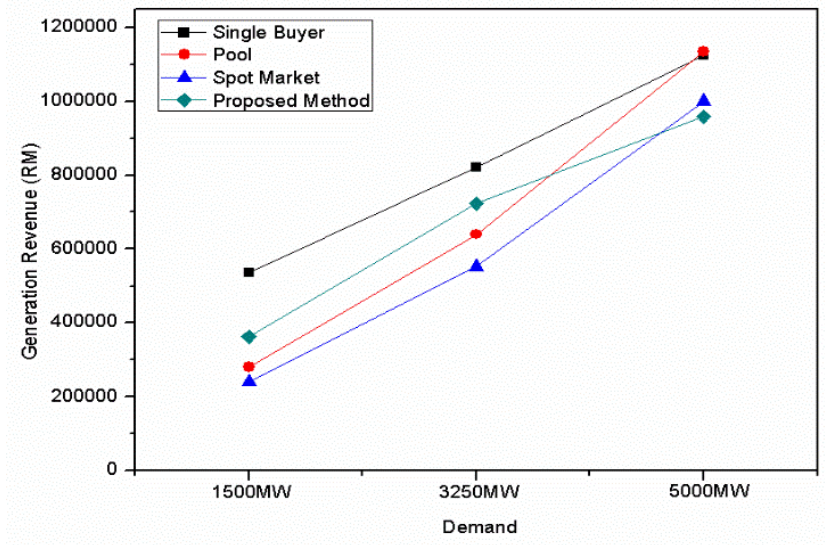

Figure 6. Comparison of total generation revenue based on demand

Table 5 shows the percentages improvement and reduction in terms of generation revenue, considering the single buyer as a base. The sign of negative data indicates the reduction percentages of generation revenue. Based on percentages, the revenue for pool and spot market models reduced more than the proposed model. During peak demand, the revenue for the pool model is slightly higher compared to the single buyer model due to high SMP. Meanwhile, the revenue for the proposed model reduced more compared to the spot market during peak demand due to the base load sharing approach which lessened market power exercises. The reduced percentages indicate the improvement in lowering the demand payment. Even though the pool and spot market models show the reduction in percentages of generation revenue significantly, but still cannot guarantee reasonable income for expensive generators compared to the proposed model during low demand. The base load demand sharing approach in the proposed model has equal opportunities to participate in the trading and receive revenue for their contribution and guarantees the participation of all IPPs in the hourly trading period excluding the highest price generator.

Table 5. Percentages improvement and reduction in term of generation revenue

\begin{tabular}{cccc}
\hline Demand (MW) & Pool & Spot Market & Proposed Method \\
\hline 1500 & -47.72 & -55.26 & -32.32 \\
3250 & -22.11 & -32.76 & -12.06 \\
5000 & 0.86 & -11.08 & -14.82 \\
\hline
\end{tabular}




\subsection{Demand payment}

Figure 7 shows shows the comparison of the analysis for the demand investigation. This demand investigation is to analyse the amount of electricity generated to be paid by the purchasers to the power producers. Considering the single buyer as a base, the payment to be made for pool, spot market and proposed models decreased by $17.24 \%, 27.8 \%$ and $17.69 \%$, respectively. Even though the demand payment for spot market model is the lowest which benefit the energy buyer, this model cannot guarantee any revenue for expensive generators during low demand. The proposed model not only considers the efficiency and energy price offered by the generators, also the demand payment is reasonable to the purchasers.

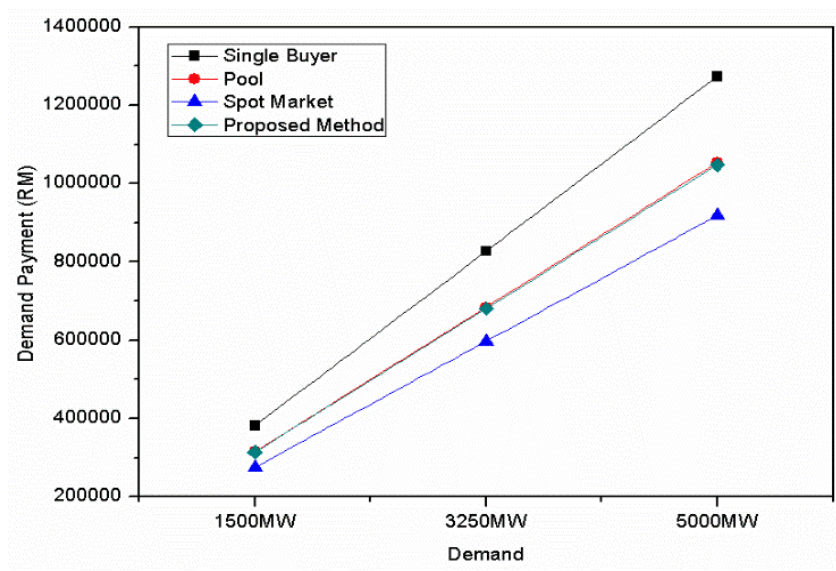

Figure 7. The analysis of the demand side investigation

\section{CONCLUSION}

Under the single buyer model, the generators had gained the largest revenue due to the existence of both capacity and energy payment. These generators still can obtain revenue even without any contribution to supply the load demand. This model does not provide any competition due to the long-term agreement; that simplify the electricity trading under one company which is TNB transmission and distribution. The pool market model offers full competitive model and based on uniform price scheme. This model fully removed the capacity payment and therefore reduces the revenue some of the generators quite significantly. The most expensive generators might not be able to get any revenue at all and hence will force each of them to bid for the cheapest energy price most of the time and this will create competition. Overall, the pool and spot market models show similar characteristic.

From the observation, the result indicates that the proposed model managed to overcome the problem of the pool model due to low or no revenue during low demand and price fluctuations during high demand which caused power market exercise. The proposed model still maintained the capacity payment in a fair manner which benefit the generators without burdening the purchasers with high price energy. The proposed model gives the opportunity more to the least cost generators to participate not only in base load demand but also in peak load demand. The minimum generation capacity payment also been given as an incentive and compensation for the least cost and expensive generator, which participate and lost in bidding competition respectively, it create win-win situation between the generators as the seller and distributor as the buyer.

\section{ACKNOWLEDGEMENTS}

The authors gratefully appreciate the Universiti Tun Hussein Onn Malaysia (UTHM) for supporting this research work under TIER 1 grant vot H156.

\section{REFERENCES}

[1] S. Gorgizadeh, Akbari Foroud, A., and Amirahmadi, M., "Strategic bidding in a pool-based electricity market under load forecast uncertainty," Iranian Journal of Electrical and Electronic Engineering, vol. 8, pp. 164-176, 2012.

[2] J. Huang, Y. Xue, Z. Y. Dong and K. P. Wong, "An Efficient Probabilistic Assessment Method for Electricity Market Risk Management," in IEEE Transactions on Power Systems, vol. 27, no. 3, pp. 1485-1493, Aug. 2012. 
[3] N. Othman, M.Y. Hassan, F. Hussin, and M. P. Abdullah, "Generator Revenue Adequacy in the Competitive Electricity Markets: The case of Malaysia," International Journal of Integrated Engineering, vol. 5, pp. 26-35, 2013.

[4] N. Z. Mohd Zamin, N. Z. Zainol Abidin and J. B. Ibrahim, "Single buyer - A step forward in Malaysian Electricity Supply Industry reform," IEEE 2013 Tencon - Spring, Sydney, NSW, 2013, pp. 391-397.

[5] M. F. Yusop and N. Y. Dahlan, "Study of capacity payment prices for IPPs in Malaysia," 2014 IEEE 8th International Power Engineering and Optimization Conference (PEOCO2014), Langkawi, 2014, pp. 335-340.

[6] N. Mohamad and P. D. A. Aziz, "Evaluation of economic benefits for market models in restructured electricity supply industry (ESI)," 2014 4th International Conference on Engineering Technology and Technopreneuship (ICE2T), Kuala Lumpur, 2014, pp. 28-32.

[7] M. Y. Hassan, M. P. Abdullah, A. S. Arifin, F. Hussin and M. S. Majid, "Electricity market models in restructured electricity supply industry," 2008 IEEE 2nd International Power and Energy Conference, Johor Bahru, 2008, pp. 1038-1042.

[8] Z. Ngadiron and N. H. Radzi, "Generation revenue assessment on restructuring the Malaysia electricity supply industry," ARPN Journal of Engineering and Applied Sciences, vol. 11, no. 6, pp. 3805-3811, March 2016.

[9] R. N. Allan and H. Navarro Sanchez, "Uncertainty considerations in the pool purchase price in the England and Wales electricity supply industry," in IEE Proceedings-Generation, Transmission and Distribution, vol. 141, no. 2, pp. 125-132, March 1994.

[10] F. Olsina, R. Pringles, C. Larisson, and F. Garcés, "Reliability payments to generation capacity in electricity markets," Energy Policy, vol. 73, pp. 211-224, 2014.

[11] N. Othman, M. Y. Hassan and F. Hussin, "Generation revenue assessment in pool-based electricity markets," 2012 IEEE International Conference on Power and Energy (PECon), Kota Kinabalu, 2012, pp. 206-211.

[12] H. Outhred, "The competitive market for electricity in Australia: why it works so well," Proceedings of the 33rd Annual Hawaii International Conference on System Sciences, Maui, HI, USA, 2000, pp. 8

[13] A. Zahedi and M. Aldeen, "Evaluation of PV solar power generation scenarios in reducing electricity spot price during hot summer days in Victoria, Australia," 2014 Australasian Universities Power Engineering Conference (AUPEC), Perth, WA, 2014, pp. 1-6.

[14] M. S. Smith, and T. S. Shively, "Econometric modeling of regional electricity spot prices in the Australian market," Energy Economics, vol. 74, pp. 886-903, 2018.

[15] K. Ignatieva, and S. Trück, "Modeling spot price dependence in Australian electricity markets with applications to risk management," Computers \& Operations Research, vol. 66, pp. 415-433, 2016.

[16] Australia Electricity Market Operator, "An introduction to Australia's National Electricity Market," 2010.

[17] A. Moran, and R. Sood, "Chapter 19 - Evolution of Australia's National Electricity Market," Evolution of Global Electricity Markets, Academic Press, pp. 571-614, 2013.

[18] M. Jeppesen, M.J. Brear, D. Chattopadhyay, C. Manzie, R. Dargaville, and T. Alpcan, "Least cost, utility scale abatement from Australia's NEM (National Electricity Market). Part 1: Problem formulation and modelling," Energy, vol. 101, pp. 606-620, 2016.

[19] Z. Ngadiron, N. H. Radzi and M. Y. Hassan, "The generation revenue and demand side assessment in pool-based market model for competitive electricity markets," 2016 IEEE International Conference on Power and Energy (PECon), Melaka, 2016, pp. 372-377.

[20] Z. Ngadiron, N. H. Radzi, and M. Y. Hassan, "The economic benefits of generation revenue assessment in poolbased market model for restructured electricity supply industry," presented at 2nd International Conference on Green Design and Manufacture, Phuket, Thailand, MATEC Web Conf, vol. 78, 2016.

[21] N. Y. Dahlan, M. S. Amran, Y. A. Kushairi and H. Mohamad, "Competitive bidding prices for new generation capacity in Malaysia considering uncertainty," 2013 IEEE 3rd International Conference on System Engineering and Technology, Shah Alam, 2013, pp. 215-220.

[22] Z. Ngadiron, and N. H. Radzi, "Feed-in-tariff and competitive auctions as support mechanism for renewable energy: a review," ARPN Journal of Engineering and Applied Sciences, vol. 11, no. 14, pp. 8938-8946, July 2016.

[23] Z. Ngadiron, N. H. Radzi, M.Y. Hassan, and R. C. Bansal, "The economic benefits of generation revenue and demand payment assessment in pool-based market model: The case of Malaysia," Electric Power Components and Systems, vol. 46, no. 1, pp. 56-68, 2018

[24] I. E. A. Energy Technology Network, "Gas-fired power, energy technology system analysis programme," IEA ETSAP-Technology Brief E02, 2010.

[25] P. Vithayasrichareon and I. F. MacGill, "Impacts of generation-cycling costs on future electricity generation portfolio investment," 2014 IEEE PES General Meeting|Conference \& Exposition, National Harbor, MD, 2014, pp. 1-5. 


\section{BIOGRAPHIES OF AUTHORS}
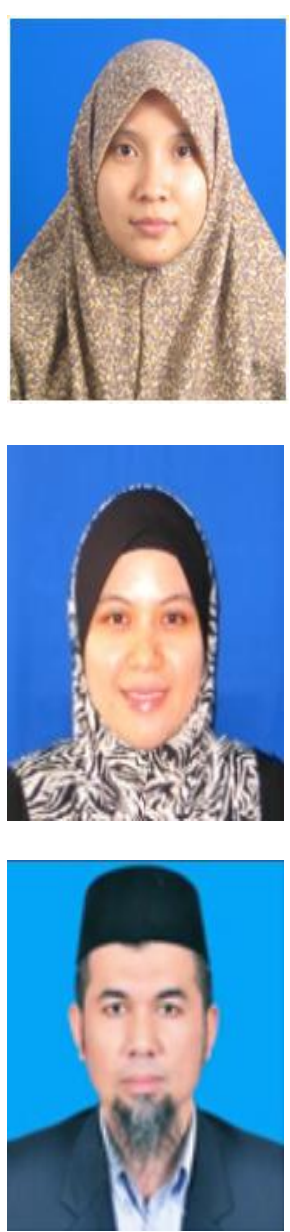

Zuraidah Ngadiron received the B.Eng. degree from Universiti Teknologi Malaysia Johor, Malaysia, in 2002, and the M.E.E. degree and Ph.D degree from University Tun Hussein Onn Malaysia, in 2008 and 2018, respectively. She is currently a research assistant at Universiti Tun Hussein Onn Malaysia. Her research interests are in power system economics, transmission pricing, energy management, and renewable energy.

Nur Hanis Radzi received the B.Eng. and the M.E.E. degree from Universiti Teknologi Malaysia, Johor, Malaysia, in 2005 and 2009, respectively, and the Ph.D. degree from Universiti of Queensland, Australia, in 2012. She is currently a lecturer at University Tun Hussein Onn Malaysia. Her research is focused on power system economics, transmission pricing, energy management, and renewable energy.

Mohammad Yusri Hassan received the B.Eng. degree in electrical and electronics engineering from Strathclyde Universiti, U.K., in 1988, the M.E.E. degree from Universiti Teknologi Malaysia, Johor, Malaysia, in 1993, and the Ph.D. degree from Strathclyde Universiti, U.K., in 2004. He is a Professor and Director of Centre of Electrical Energy Systems (CEES) in the Faculty of Electrical Engineering at UTM. His research interests are in power system economics, transmission pricing, and energy management. 